FTUV/94-34

\title{
A novel kind of neutrino oscillation experiment
}

\author{
J. Segura, J. Bernabéu, F.J. Botella and J. A. Peñarrocha \\ Departament de Física Teòrica \\ Universitat de València \\ and \\ IFIC, Centre Mixt Univ. València-CSIC \\ E-46100 Burjassot, Spain
}

\begin{abstract}
A novel method to look for neutrino oscillations is proposed based on the elastic scattering process $\bar{\nu}_{i} e^{-} \rightarrow \bar{\nu}_{i} e^{-}$, taking advantage of the dynamical zero present in the differential cross section for $\bar{\nu}_{e} e^{-} \rightarrow \bar{\nu}_{e} e^{-}$. An effective tunable experiment between the "appearance" and "disappearance" limits is made possible. Prospects to exclude the allowed region for atmospheric neutrino oscillations are given.
\end{abstract}


Three aspects are essential in a neutrino oscillation [1] experiment: the source, the evolution ( to allow oscillations ) and the detection. If we restrict ourselves to flavour vacuum oscillations, essentially two types of experiments have been proposed and performed : the so called appearance and disappearance [2] limits. In an appearance experiment a certain source produces a neutrino of a given flavour and, after evolution, the experiment tries to detect neutrinos of another flavour. Since flavour is defined through charged current interactions it is customary to use a pure charged current interaction to detect the new flavour. Charged current detection has a threshold for production so that it is impossible to use low energy electron neutrinos ( or antineutrinos ) for appearance experiments. In particular, it must be stressed that the copious reactor antineutrinos cannot be used for appearance experiments. This is certainly a drawback to explore regions in the $\Delta m^{2}-\sin ^{2} 2 \phi$ plane where both $\Delta m^{2}$ ( the difference of neutrino squared masses ) and $\sin ^{2} 2 \phi$ ( $\phi$ being the mixing angle) are small. Note that the use of low energy neutrinos implies a better sensitivity to low $\Delta m^{2}$ for a given distance from the source to the detector. In general, appearance experiments are more sensitive to small mixing angles than the disappearance ones.

In disappearance experiments there is a controlled source which produces a given flavour and a detector which sees the same flavour via a charged current interaction with some target: a depletion in the flux after neutrino travelling would be a manifestation of oscillation. For small mixing, however, the dominant signal in the detector comes from neutrinos with the original flavour, so this translates into less sensitivity to small mixing angles in disappearance experiments.

As a consequence we see that, for example, in order to explore in the laboratory the region of masses and mixing where potential neutrino oscillations from atmospheric neutrinos [3] have been suggested it is necessary to consider detectors very far away from the source [4] with the corresponding reduction in the flux.

For $\nu_{e}$ and $\bar{\nu}_{e}$ beams the previous arguments are true provided the detection reaction is a pure charged current one. We are going to consider a mixed charged and neutral current reaction like $\bar{\nu}_{e} e^{-} \rightarrow \bar{\nu}_{e} e^{-}$, making use of the fact that the corresponding cross section is different from the one for $\bar{\nu}_{\mu} e^{-} \rightarrow \bar{\nu}_{\mu} e^{-}$, which only has a neutral current contribution. In principle, it could be possible to perform a neutrino oscillation experiment $\left(\bar{\nu}_{e} \rightarrow \bar{\nu}_{\mu}\right)$ just by measuring the cross section for the scattering of neutrinos on electrons at some distance from the neutrino source. If oscillations 
take place some component of the beam will change, for example, from $\bar{\nu}_{e}$ to $\bar{\nu}_{\mu}$, and the total number of recoil electrons detected will be different from the counting one would have if no oscillations occurred. However the fact that both total cross sections are similar ( at high energies the electron antineutrino total cross section is about 3 times larger that the corresponding one for muon antineutrino ) seems to disfavour this possibility of studying oscillations.

Nevertheless, recently it has been proved [5] that the cross section for the scattering of electron antineutrinos on electrons has a dynamical zero for the kinematical configuration corresponding to maximum electron recoil energy $T$ for an incident antineutrino energy $E_{\nu}=m_{e} /\left(4 \sin ^{2} \theta_{W}\right) \simeq m_{e}$, being $m_{e}$ the electron mass. This zero is not present in $\bar{\nu}_{\mu} e^{-} \rightarrow \bar{\nu}_{\mu} e^{-}$. So it is possible to imagine an experiment with an ideal monoenergetic beam with $E_{\nu} \simeq m_{e}$ measuring the cross sections with recoil electrons at $T=T_{\max }=2 E_{\nu}^{2} /\left(2 E_{\nu}+m_{e}\right) \simeq 2 m_{e} / 3$. If any signal is found it would come from $\bar{\nu}_{\mu} e^{-} \rightarrow \bar{\nu}_{\mu} e^{-}$after the initial $\bar{\nu}_{e}$ has oscillated to $\bar{\nu}_{\mu}$.

In the standard theory the differential cross section for the process $\bar{\nu}_{i} e^{-} \rightarrow \bar{\nu}_{i} e^{-}$ [6] is given by

$$
\frac{d \sigma^{\bar{\nu}_{i}}}{d T}=\frac{2 G^{2} m_{e}}{\pi}\left[\left(g_{R}^{i}\right)^{2}+\left(g_{L}^{i}\right)^{2}\left(1-\frac{T}{E_{\nu}}\right)^{2}-g_{L}^{i} g_{R}^{i} \frac{m_{e} T}{E_{\nu}^{2}}\right]
$$

where $G$ is the Fermi coupling constant, $T$ the recoil kinetic energy of the electron and $E_{\nu}$ the antineutrino incident energy. For $\nu_{i}$ one has to make the change $g_{L}^{i} \leftrightarrow g_{R}^{i}$ in Eq. (1). In terms of the weak mixing angle $\theta_{W}$, the chiral couplings $g_{L}^{i}$ and $g_{R}^{i}$ can be written for each neutrino flavour as

$$
\begin{array}{cc}
g_{L}^{e}=\frac{1}{2}+\sin ^{2} \theta_{W}, & g_{R}^{e}=\sin ^{2} \theta_{W} \\
g_{L}^{\mu, \tau}=-\frac{1}{2}+\sin ^{2} \theta_{W}, & g_{R}^{\mu, \tau}=\sin ^{2} \theta_{W}
\end{array}
$$

From Eq. (1) it is evident that if $g_{L}^{i} g_{R}^{i}>0$ there is a chance for the cross section to cancel in the physical region. From Eq. (2) we see that this zero is only possible in the $\bar{\nu}_{e} e^{-} \rightarrow \bar{\nu}_{e} e^{-}$channel and, in fact, it takes place for the kinematical configuration $E_{\nu}=m_{e} /\left(4 \sin ^{2} \theta_{W}\right)$ and maximal $T$. Neither $d \sigma^{\bar{\nu}_{\mu}} / d T$ nor $d \sigma^{\bar{\nu}_{\tau}} / d T$ present a dynamical zero since $g_{L}^{\mu, \tau} g_{R}^{\mu, \tau}<0$. We will take advantage of this fact to propose a novel kind of neutrino oscillation experiment for reactor antineutrinos. It is important to stress several additional facts which explain why it is worthwhile to 
study more carefully this sort of "appearance" experiment using a neutral current reaction for neutrino "detection":

i) The dynamical zero is only present for $\bar{\nu}_{e}$, not for $\nu_{e}$ or $\nu_{\mu}\left(\bar{\nu}_{\mu}\right), \nu_{\tau}\left(\bar{\nu}_{\tau}\right)$.

ii) The flavour $\bar{\nu}_{e}$ is precisely the one which is produced copiously in nuclear reactors.

iii) The neutrino energy at which the zero appears is on the peak of the antineutrino reactor spectrum [6, 7].

iv) The dynamical zero is located at the maximum electron recoil energy $T \simeq 2 m_{e} / 3$. This value is in the range of the proposed experiments [8, 9, 10] to detect recoil electrons.

v) In spite of the fact that the antineutrino reactor spectrum is continuous, there are several proposals with detectors that will be able to select the incident neutrino energies by measuring both the electron recoil energy and its recoil angle

vi) Last but not least, the use of $0.5 \mathrm{MeV}$ reactor antineutrinos in this sort of appearance experiment would imply a good sensitivity to rather low $\Delta m^{2}$ values. In particular, as we will see, this kind of experiment would be sensitive to the region in the $\Delta m^{2}-\sin ^{2} 2 \phi$ plane where potential oscillations have been suggested from atmospheric neutrinos.

The purpose of this paper consists in studying the potentialities of using the detection reaction $\bar{\nu}_{i} e^{-} \rightarrow \bar{\nu}_{i} e^{-}$in a neutrino oscillation experiment, taking advantage of the dynamical zero present in the cross section for $\bar{\nu}_{e} e^{-} \rightarrow \bar{\nu}_{e} e^{-}$.

Suppose we have a source of electron-antineutrinos $\bar{\nu}_{e}(0)$ ( a nuclear reactor for example ) and we measure the differential cross section for the process $\bar{\nu}_{e}(x) e^{-} \rightarrow$ $\bar{\nu}_{e}(x) e^{-}$at a distance $x$ from the source. If vacuum oscillations take place we will have

$$
\frac{d \sigma^{\bar{\nu}}\left(E_{\nu}, T, x\right)}{d T}=P_{\bar{\nu}_{e} \rightarrow \bar{\nu}_{e}}(x) \frac{d \sigma^{\bar{\nu}_{e}}\left(E_{\nu}, T\right)}{d T}+\sum_{i=\mu, \tau} P_{\bar{\nu}_{e} \rightarrow \bar{\nu}_{i}}(x) \frac{d \sigma^{\bar{\nu}_{i}}\left(E_{\nu}, T\right)}{d T}
$$

where $P_{\bar{\nu}_{e} \rightarrow \bar{\nu}_{i}}(x)$ is the probability of getting a $\bar{\nu}_{i}$ at a distance $x$ form the source. Taking advantage of the conservation of probability ( we disregard oscillation to sterile neutrinos ) and the identity $d \sigma^{\bar{\nu}_{\mu}} / d T=d \sigma^{\bar{\nu}_{\tau}} / d T$, Eq. (3) can be written as 


$$
\frac{d \sigma^{\bar{\nu}}\left(E_{\nu}, T, x\right)}{d T}=\frac{d \sigma^{\bar{\nu}_{e}}\left(E_{\nu}, T\right)}{d T}+\left(\frac{d \sigma^{\bar{\nu}_{\mu}}\left(E_{\nu}, T\right)}{d T}-\frac{d \sigma^{\bar{\nu}_{e}}\left(E_{\nu}, T\right)}{d T}\right) \sum_{i=\mu, \tau} P_{\bar{\nu}_{e} \rightarrow \bar{\nu}_{i}}(x)
$$

In the particular case of considering only two flavour oscillation we have:

$$
\sum_{i=\mu, \tau} P_{\bar{\nu}_{e} \rightarrow \bar{\nu}_{i}}(x) \rightarrow P_{\bar{\nu}_{e} \rightarrow \bar{\nu}_{\mu}}(x)=\sin ^{2} 2 \phi \sin ^{2}\left(\frac{\Delta m^{2} x}{4 E_{\nu}}\right)
$$

where $\phi$ is the vacuum mixing angle and $\Delta m^{2}$ is the difference of the square of masses of the mass eigenstates $\nu_{1}$ and $\nu_{2}$.

From Eq. (4) it is quite evident that by measuring $d \sigma^{\bar{\nu}} / d T$ at the kinematical configuration where $d \sigma^{\bar{\nu}_{e}} / d T$ vanishes, the signal will be proportional to the oscillation probability times the $\bar{\nu}_{\mu} e^{-} \rightarrow \bar{\nu}_{\mu} e^{-}$cross section thus simulating an "appearance" experiment. Moving to different configurations one has the possibility to tune the relative contribution of the two terms of Eq.(4) and thus the sensitivity to $\sin ^{2} 2 \phi$ and $\Delta m^{2}$. It must be stressed that Eq. (4) is a valid description for the neutrinoelectron cross section for any energy $E_{\nu}$ and $T$ and so in principle it could be used with any source of electron antineutrinos. Advantage must be taken from this dependence on $E_{\nu}$ and $T$ in order to select the appropriate kinematical region in each type of experiment. On top of the dynamical zero our observable is a pure "appearance" experiment ,outside the zero it is a mixture of "appearance" and "disappearance"; the observable could simulate a pure disappearance experiment if there were regions where $\frac{d \sigma^{\bar{\nu} e}}{d T}>>\frac{d \sigma^{\bar{\nu}} \mu}{d T}$ but this is not the case.

With high energy neutrinos $\left(E_{\nu}>>m_{e}\right)$, the $\overline{\nu_{e}}$ cross section is larger that the $\overline{\nu_{\mu}}$ and, in addition, semileptonic charged current reactions ( when possible) are much more copious. So, unless a great precision could be reached, the use of these detection reactions with high energy neutrinos does not look the most appealing.

Therefore we shall concentrate in studying the potentialities of the observable described in Eq. (4) near the dynamical zero for electron antineutrinos . This in turn means we will restrict ourselves to electron antineutrino beams coming from nuclear reactors.

In order to select the appropriate neutrino energy $E_{\nu}$ from a continuous spectrum, it is necessary to measure the electron recoil angle $\theta$ and the electron recoil energy $T$. 
To a different extent, this can be done in the proposed experiments such as MUNU [8], BOREXINO [9] and HELLAZ [10]. The kinematical relation to be used is

$$
\cos \theta=\frac{T}{\sqrt{T^{2}+2 m_{e} T}}\left(1+\frac{m_{e}}{E_{\nu}}\right)
$$

With the aim of analyzing the more convenient phase space regions for the measurement of Eq. (4) we present in Fig. 1 the curves for constant values of $d \equiv$ $\log \left[\frac{d \sigma^{\bar{\nu}} \mu}{d T} / \frac{d \sigma^{\bar{\nu} e}}{d T}\right]$ (solid lines) in the plane $(\theta, T)$. Of course the curve $d=\infty$ collapses to the point $T \simeq 2 m_{e} / 3$ and $\theta=0$, equivalent to $E_{\nu} \simeq m_{e}$ and $T=T_{\max } \simeq 2 m_{e} / 3$.

From Fig. 1 it is evident that the width of the peak in the ratio $\frac{d \sigma^{\bar{\nu}} \mu}{d T} / \frac{d \sigma^{\bar{\nu}} e}{d T}$ owing to the dynamical zero is relatively large in both directions $\theta$ and $T$. For example in the window $0.2<T<0.6 \mathrm{MeV}$ and $\theta<0.2 \mathrm{rad}$ the muon antineutrino cross section is at least 4 times bigger than the corresponding electron-antineutrino one. This kind of window enters in the capabilities of the previously mentioned experiments.

In Fig. 1, we have also plotted (dashed lines) the curves of constant $E_{\nu}$. As we will show later these lines will be useful to cancel uncertainties coming from a poor knowledge of the antineutrino spectrum [6, 7].

For the moment, let us suppose that the antineutrino spectrum from the reactor is known. In Fig. 2 we present a plausible neutrino spectrum to be used in the rest of the paper .

By inspection of Fig. 1 and Eq. 4, we see that our observable will be more sensitive to $\Delta m^{2}$ and $\phi$ in regions where $d$ is bigger, so the strategy we propose is to select events inside a region where $d$ is bigger than a certain number.

As an illustration, if we calculate the number of events in terms of $\Delta m^{2}$ and $\phi$ in the region $d>\log (5) \simeq 0.7$, take the ratio to the number of events in the absence of oscillations and impose this ratio to be $1 \pm 0.5$, the would-be exclusion plot we get is represented in Fig. 3. We have considered that the detector is placed 20 meters away from the reactor. Inside the excluded region we have inserted the by now allowed region of oscillations coming from atmospheric neutrino experiments. Taking into account the original MUNU proposal, the numbers we have considered correspond roughly to detect a few $(\sim 10)$ events per year if no oscillations take place.

If instead of taking the window $d>\log (5)$ we consider bigger windows, $d>\log (4)$ for example, there are two effects which operate in opposite sense. Let us suppose that 
the precision of the ratio \# (events with oscillations)/ \# (events without oscillations) goes as $1 / \sqrt{N}$, where $N$ is the number of detected events. Then if we integrate over $d>\log (4)$ we will have more events than with $d>\log (5)$ and so higher precision. However, we are including regions where $d$ is smaller in such a way that the second piece in the right hand side of Eq. (4) is less important and so the observable defined in this equation will be less sensitive to $\Delta m^{2}$ and $\phi$. If only these facts are taken into account we have checked that in order to exclude the atmospheric neutrino region one gets similar results if the window chosen is $d>\log (1), \log (2), \ldots \log (5)$. We do not consider higher $d$ to avoid having too few events.

These results clearly show the feasibility of this kind of experiment with the proposed detectors previously mentioned and establish the interest of the proposal in order to study the region in the $\Delta m^{2}-\phi$ plane where potential neutrino oscillations have been reported from atmospheric neutrino data. These conclusions have been obtained under the assumption of a known neutrino flux. Note that the total number of events in a given kinematical region depends on both the weak cross section at the distance $x$ and the antineutrino spectrum. So, in order to get a precision measurement of the cross section it is necessary either to measure the antineutrino spectrum in the same experiment or to reduce to a minimum the uncertainties coming from the poor knowledge of the neutrino spectrum, specially around $E_{\nu} \simeq 0.55 \mathrm{MeV}$.

The way to measure the neutrino flux in the same experiment looks theoretically very simple. One just has to measure the number of events in a kinematical region where the dependence on $\Delta m^{2}$ and $\phi$ disappears. Looking at Eq. (4) it is evident that this kinematical region is precisely the region where $d \sigma^{\bar{\nu}_{\mu}} / d T=d \sigma^{\bar{\nu}_{e}} / d T$ which corresponds to the curve $d=\log (1)=0$ in Fig. 1. So we propose to measure the number of events in the region $d=0$ where the cross section is given by

$$
\left.\frac{d \sigma^{\bar{\nu}}}{d T}\left(E_{\nu}, T, x\right)\right|_{d=0}=\left.\frac{d \sigma^{\bar{\nu}_{e}}}{d T}\left(E_{\nu}, T\right)\right|_{d=0}
$$

So by dividing the number of events in the region $d=0$ by the well known cross section in Eq. (7) one gets directly the neutrino flux. It is interesting to note that the cross section in Eq. (7) is also independent of $x$ and so it could be very useful in the calibration of the detectors in an experiment with two detectors placed at different values of $x$. 
Depending on the actual setup of an experiment, it may be that only a few events around the $d=0$ zone could be detected; then, it would be necessary to use all the available information and not just the events around $d=0$. So let us construct the following observable

$$
R_{c}=\frac{N(c)}{N(\Delta c)}
$$

where $N(c)$ is the number of detected events in the region $d>\log (c)$ and $N(\Delta c)$ the total number of events inside the region $0<\theta<0.5$ radians and $E_{\nu}^{\min }(c)<E_{\nu}<$ $E_{\nu}^{\max }(c)$ where $E_{\nu}^{\min }(c)$ and $E_{\nu}^{\max }(c)$ are the minimum and maximum values of $E_{\nu}$ for the boundary region $d \geq \log (c)$. By choosing the $E_{\nu}$ boundaries of $\Delta c$ in this way, the same part of the spectrum enters in the numerator and in the denominator of $R_{c}$ in such a way that the uncertainties tend to cancel. The $\theta$ boundary in $\Delta c$ could be chosen , depending on the final setup of the experiment, in a different way. We have checked that when we change the spectrum of Fig. 2 by a $50 \%$ in the region $E_{\nu} \leq 1.5 \mathrm{MeV}$ the error introduced in $R_{c}$ is, for $c=5$, of order $10 \%$ and so much smaller than the $50 \%$ precision needed to draw the exclusion plot in Fig. 3. For $c=5$ the window in $T$ corresponds to the reasonable range $0.1<T<1.25 \mathrm{MeV}$. So we can conclude that without including any systematic errors such as geometrical acceptances and so on, the uncertainties coming from the neutrino spectrum can be put under control either by measuring the spectrum or by the use of ratios of the kind presented in Eq. (8).

In conclusion, we have presented a novel kind of neutrino oscillation experiment based on the neutral current reaction $\bar{\nu}_{i} e^{-} \rightarrow \bar{\nu}_{i} e^{-}$, where advantage from the dynamical zero present in the cross section for $\bar{\nu}_{e} e^{-} \rightarrow \bar{\nu}_{e} e^{-}$has been taken. This kind of experiment is in general a mixture of "appearance" and "disappearance" experiments. For antineutrinos coming from a nuclear power plant and using detectors capable of measuring both the recoil energy of the electron and its recoil angle (MUNU, BOREXINO, HELLAZ ) this kind of experiment could in principle be sensitive to the by now allowed region of neutrino oscillation coming from atmospheric neutrino data.

\section{ACKNOWLEDGEMENTS}

This paper has been supported by CICYT under Grant AEN 93-0234. We are indebted to G. Bonvicini, C. Broggini, J. Busto, J.F. Cavaignac, M. Giammarchi and 
D.H. Koang for discussions about the topic of this paper. 


\section{References}

[1] B. Pontecorvo, Sov. Phys. JETP 6 (1958) 429,

S.M. Bilenky and B. Pontecorvo, Phys. Rep. 41 (1978) 225.

[2] For a review of both types of experiments before 1987 see S.M. Bilenky and S. Petcov, Rev. Mod. Phys. 59(1987)671; see also A. Yu. Smirnov,"Results from neutrino experiments", talk given at the International Symposium "LeptonPhoton interactions", Cornell University, Ithaca, New York, August 1993, preprint IC/93/388 and J-P. Revol, "Future neutrino oscillation experiments", presented at the "Rencontrés de Physique de la Valle d'Aoste 1993", Preprint CERN-PPE/93-84.

[3] K.S Hirata et al. (Kam-II collaboration), Phys. Lett B280(1992)146,

R. Becker-Szendy et al. (IMB Collaboration), Phys. Rev. D46(1992)3720,

Ch. Berger et al. (Fréjus Collaboration), Phys. Lett. B245(1990)305, Phys. Lett. B227 (1989)489,

E. Kh. Akhmedov, "Atmospheric Neutrinos", preprint FTUV/94-9, IFIC/94-6.

[4] S. Parke in Proceedings of the XXXVIIIth Rencontré de Moriond (1993), "Perspectives in Neutrinos, Atomic Physics and Gravitation", eds.: J. Trân Thanh Vân, T. Damour, E. Hinds and J. Wilkerson, pg. 229;

N.C. Mascarenhas, ibidem, pg. 237.

[5] J. Segura, J. Bernabéu, F.J. Botella and J. Peñarrocha, Phys. Rev. D49(1994)1633.

[6] P. Vogel and J. Engel, Phys. Rev. D39(1989)3378.

[7] H. V. Klapdor and J. Metzinger, Phys. Lett. B112 (1982) 22,

K. Schreckenbach et al., Phys. Lett. B160 (1985) 325,

O. Tengblad et al., Nucl. Phys. A503(1989) 136.

[8] C. Broggini et al., "A detector for the study of $\bar{\nu}_{e} e^{-}$at a nuclear reactor", Preprint LNGS-92/47,

C. Broggini et al., Proceedings of the XXXVIIIth Rencontré de Moriond (1993),

"Perspectives in Neutrinos, Atomic Physics and Gravitation", pg. 255. 
[9] M. Giammarchi et al., contribution to TAUP'93, LNGS.

[10] G. Bonvicini et al., contribution to TAUP'93, LNGS. 


\section{Figure Captions.}

- Fig. 1) Curves for constant values of $d \equiv \log \left[\frac{d \sigma^{\bar{\nu}} \mu}{d T} / \frac{d \sigma^{\bar{\nu} e}}{d T}\right]$ (solid lines), from $d=0$ to $\mathrm{d}=2$, and for constant values of $E_{\nu}$ in $\mathrm{MeV}$ (dashed lines) in the plane $(T, \theta)$.

- Fig. 2) A plausible $\bar{\nu}_{e}$ spectrum from a nuclear reactor.

- Fig. 3) Would-be exclusion plot obtained by imposing that the ratio $\int \frac{d \sigma^{\bar{\nu}}}{d T} / \int \frac{d \sigma^{\bar{\nu}} e}{d T}$ (ratio oscillation/non-oscillation) is less than 1.5, integrating the cross sections over a typical reactor spectrum in the kinematical region where $\frac{d \sigma_{\bar{\nu}}}{d T} / \frac{d \sigma_{\bar{\nu}}}{d T} \geq 5$ and considering the detector is 20 meters away from the reactor. The shaded zone corresponds to the allowed region for atmospheric $\nu_{e} \leftrightarrow \nu_{\mu}$ oscillations. 
This figure "fig1-1.png" is available in "png" format from: http://arxiv.org/ps/hep-ph/9406305v1 
This figure "fig1-2.png" is available in "png" format from: http://arxiv.org/ps/hep-ph/9406305v1 
This figure "fig1-3.png" is available in "png" format from: http://arxiv.org/ps/hep-ph/9406305v1 